\title{
Article \\ Perpendicular exchange bias of Co/Ni multilayers adjacent to antiferromagnetic FeRh Layer
}

\author{
Soyoung Jekal ${ }^{1,2, *}$ (i) and Dao Phuong ${ }^{3}$ \\ 1 Laboratory of Metal Physics and Technology, Department of Materials, ETH Zurich, 8093 Zurich, \\ Switzerland \\ 2 Condensed Matter Theory Group, Paul Scherrer Institute, CH-5232 Villigen PSI, Switzerland \\ 4 Hefei National Laboratory, University of Science and Technology of China, Hefei, Anhui 230026, China \\ * Correspondence: so-young.jekal@mat.ethz.ch; Tel.: +4144632 2643
}

\begin{abstract}
The perpendicular exchange bias effect is observed in the ferromagnetic $\mathrm{Co} / \mathrm{Ni}$ multilayers adjacent to the antiferromagnetic FeRh layer. It is found that as the antiferromagnetic FeRh thickness increases from $10 \AA$ to $50 \AA$, the hysteresis loop is gradually changed from the symmetric shape to the asymmetric shape shifted by some amount corresponding to the exchange-biased field at the thickness of $25 \AA$. Also, the magnetic domain observation experiment confirms that the domain reversals in the increasing and the decreasing field regions of the sample with the thickness of $50 \AA$ exhibit the same single domain wall motion even though they have the different coercivities.
\end{abstract}

Keywords: exchange bias effect, perpendicular exchange bias

\section{Introduction}

In general, when the ferromagnetic (FM) film is adjacent to the antiferromagnetic (AFM) layer, the magnetic hysteresis loop of the film exhibits an asymmetric shape moved to a particular direction of magnetic field due to the interaction at the interface[1]. Such phenomena in the AFM/FM film is called as an exchange bias effect. In addition, the magnetic hysteresis loop shifted to a certain direction is used to measure the degree of the exchange bias effect. While the exchange bias effect is classified as an in-plane exchange bias and a perpendicular exchange bias depending on the magnetic anisotropy direction of the FM film, most studies have been done in the in-plane exchange bias so far[2-8]. However, since the perpendicular exchange bias is recently discovered, new phenomenon in the promising exchange bias have attracted new attention due to the possibility of application to spintronics devices such as magnetic sensors, magnetic recording read heads and magnetic random access memory (MRAM)[9-15].

In most FM/AFM structures with perpendicular exchange bias effect, $\mathrm{Co} / \mathrm{Pd}[16-18]$ and $\mathrm{Co} / \mathrm{Pt}[9-$ 11,15] mulilayers have being mainly used for the FM material due to their strong perpendicular magnetic anisotropy, while FeRh[19-21], FeMn[11,22], $\operatorname{IrMn}[9,15,23,24]$, and $\mathrm{FeCl}_{2}$ [25] are considered as the AFM material. Furthermore, since the asymmetric inversion on magnetization is observed in the perpendicular exchange bias, the magnetic domain movement also have been actively studied to a broaden understanding of the exchange bias[13,26,27]. In particular, the change of the exchange bias magnetic field $H_{E}$ and the coercivity $H_{C}$ is known as the results of the complex interaction of AFM/FM domain wall as well as an unidirectional anisotropy coming from the AFM/FM interface[1,2,28,29].

In this paper, we confirmed the perpendicular exchange bias effect experimentally in a thin film composed of FM Co/Ni multilayer and AFM FeRh layer. In recent years, Co/Ni multilayer is being used for the current-induced spin dynamics due to its relatively small $H_{C}$ and fast domain wall mobility compared to $\mathrm{Co} / \mathrm{Pd}$ and $\mathrm{Co} / \mathrm{Pt}$ multilayers[30-39]. In order to investigate the thickness of 
(a)

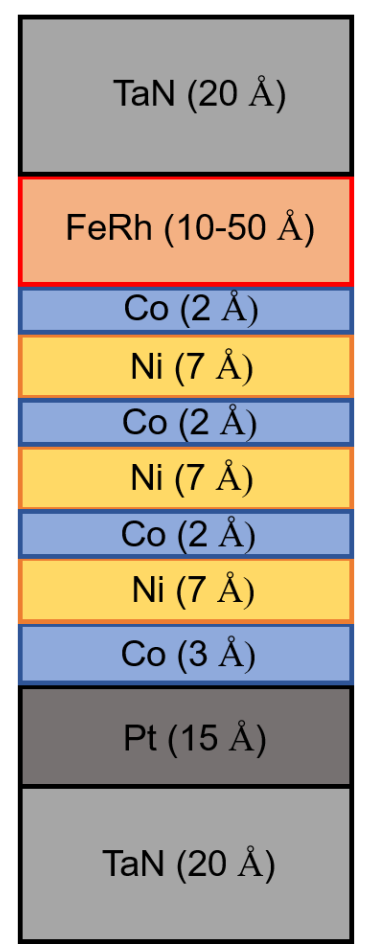

(b)

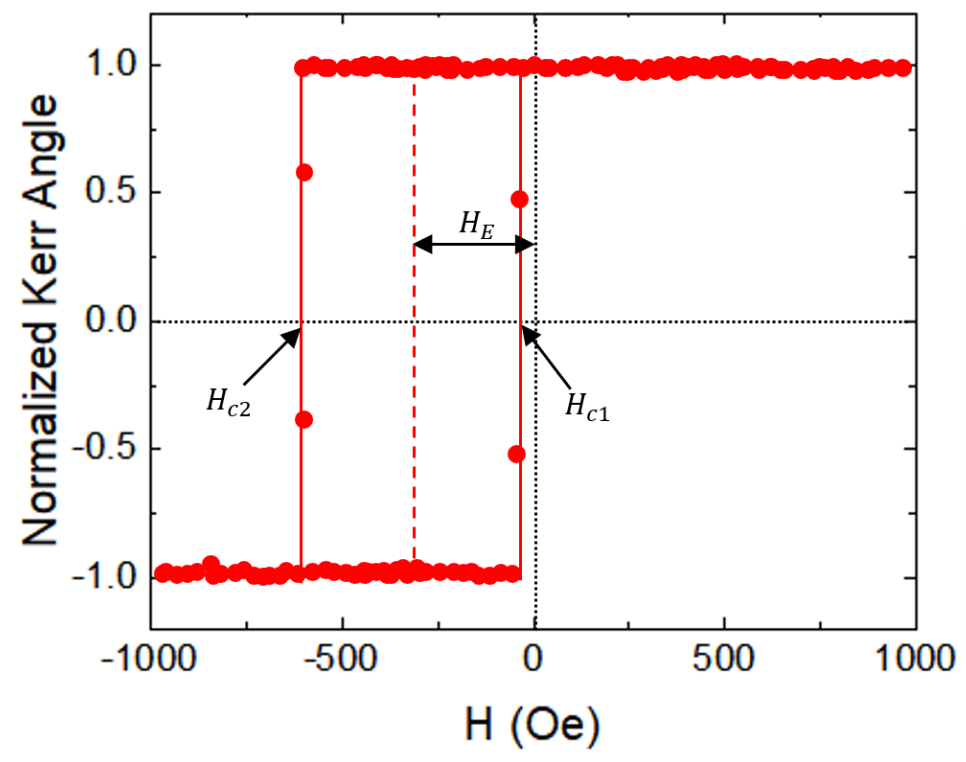

Figure 1. (a) Schematic diagram of the detailed sample structure basically consisting of the FM Co/Ni multilayer and the AFM FeRh layer. (b) Kerr hysteresis loop of the sample with the FeRh thickness of $50 \AA$ exhibiting the exchange bias effect. Here, $\mathrm{H}_{\mathrm{C} 1}$ and $\mathrm{H}_{\mathrm{C} 2}$ correspond to the coercivities in the increasing and the decreasing field regions, respectively. $\mathrm{H}_{E}$ denotes the exchange-biased field.

AFM layer dependent exchange bias effect, we produced samples with various thickness (from 10 $\AA$ to $50 \AA$ ) of FeRh which is G-type AFM material. G-type AFM material is a promising candidate to tailor the interface spin configuration for exchange bias. In G-type AFM materials, the nearest neighboring magnetic moments on the (001) plane align antiferromagnetically whereas those on the (111) plane have a ferromagnetically ordered spin configuration, providing the ideal uncompensated interface for exchange bias[40]. The magnetic hysteresis loops were measured by using a home-built longitudinal magneto-optical Kerr effect (MOKE) set-up based on photoelastic modulation. In addition, the magnetic domain revolution patterns in regions with both increasing and decreasing magnetic field were observed by using a magneto-optical microscope.

\section{Materials and Methods}

The samples were deposited on the $\mathrm{Si}(100)$ substrate by using a dc magnetron sputtering system under working pressure of $1.4 \times 10^{-6}$ Torr. The Si(100) substrates were loaded for deposition without removing the amorphous $\mathrm{SiO}_{2}$, and the targets were pre-sputtered for 20 minute before deposition. A dc-magnetic field of $1.5 \mathrm{kOe}$ was applied parallel to the substrate surface during the growth of the film to set the exchange bias direction in the films. All the layers were deposited at room temperature. During magnetic annealing was carried out in a vacuum chamber in $5 \times 10^{6}$ Torr at $400{ }^{\circ} \mathrm{C}$ for 1 hour, the annealing temperature was controlled using a proportional-integral-derivative (PID) controller.

The detailed structure of sample is illustrated in Fig. 1(a). In the exchange bias, Co/Ni multilayer with Pt contributes to the perpendicular magnetic anisotropy, while FeRh serves as AFM material. TaN deposited above and below the layer was used for the capping layer.

The measurements of the magnetic hysteresis loops were carried out using a home-built longitudinal MOKE. A solenoid was used to generate the magnetic field in steps of 0.3 Oe by sweeping the current using a programmable constant current source. The thickness of the individual layers was 
determined using specular X-ray reflectivity measurements carried out using X-pert Panalytical X-ray diffractometer. In addition, the magnetic domain revolution patterns under the increasing and the decreasing magnetic field were observed by using a magneto-optical microscope. The obtained image was converted to an artificial image with better contrast through a real-time image processing system.

\section{Results and Discussion}
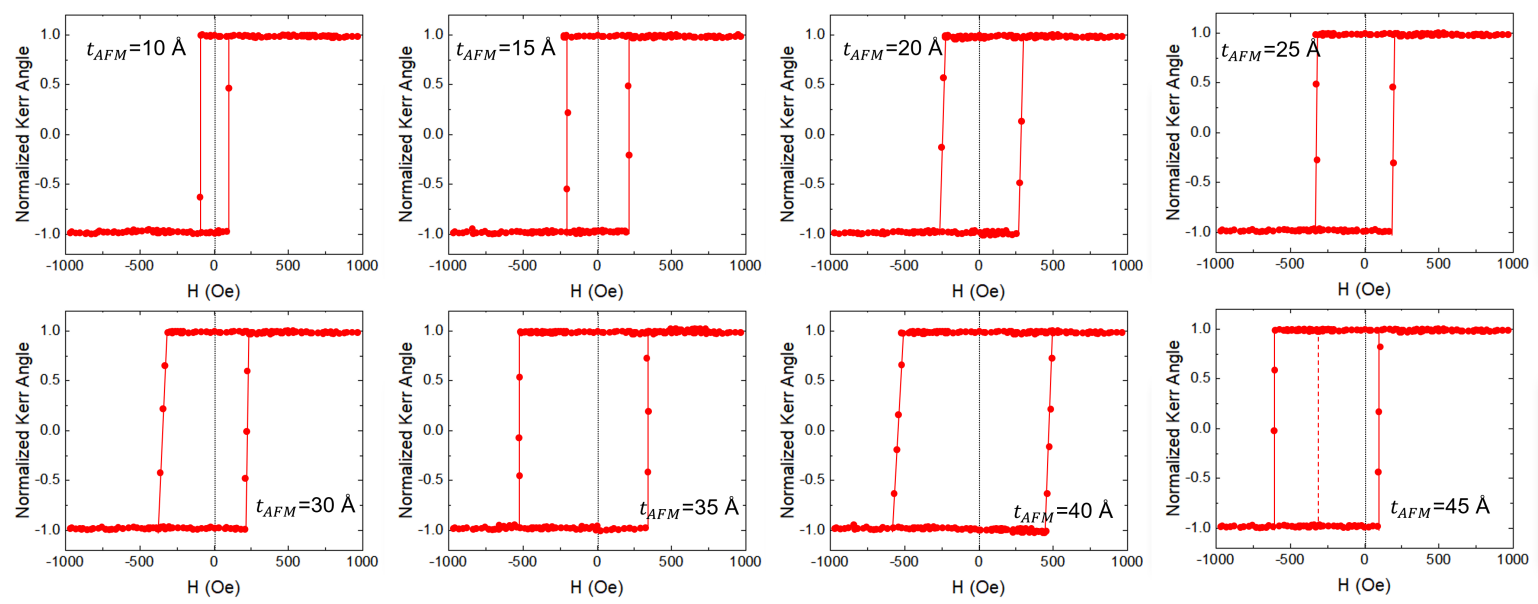

Figure 2. Various Kerr hysteresis loops of the samples with the FeRh thicknesses varying from $10 \AA$ to $45 \AA$.

Using a longitudinal MOKE, we measured the magnetic hysteresis loop of the $\mathrm{FM} \mathrm{Co} / \mathrm{Ni}$ multilayer adjacent to the AFM FeRh layer of various thicknesses. Fig. 1(b) shows a magnetic hysteresis loop of a sample having a FeRh thickness $t_{A F M}=50 \AA$. The Kerr angle corresponding to the magneto-optical intensity is almost proportional to the magnetization $M$ of the magnetic material. Therefore, we present normalized Kerr angle $\left(\theta / \theta_{S}\right)$ which corresponds to saturated magnetization $\left(M_{S}\right)$ with angle of Kerr $\theta$ for convenience. Unlike a typical ferromagnetic thin film which shows a symmetrically shaped magnetic hysteresis loop, it exhibits an asymmetric shape moved from the origin. This is due to an unidirectional-anisotropy perpendicular to the sample created by the exchange interaction between the FM Co/Ni multilayer and the AFM FeRh layer at the interface. Such unidirectional-anisotropy shows different coercivity depending on the direction of the magnetic field in the magnetic hysteresis loop, as shown in Fig. 1(b). In order to investigate the magnetic properties of the AFM/FM film indicating the exchange interaction, the magnetic coercivity $\mathrm{H}_{C}$ and the exchange bias field $\mathrm{H}_{E}$ should be measured. In particular, the $\mathrm{H}_{E}$ is an important value to predict the degree of exchange interaction between FM and AFM layers. For the Co/Ni multilayer with $50 \AA \mathrm{FeRh}, \mathrm{H}_{\mathrm{C}}$ and $\mathrm{H}_{E}$ were measured as $225 \pm 5$ and $325 \pm 5 \mathrm{Oe}$, respectively. These values were calculated from relations of $\mathrm{H}_{C}=\left(\mathrm{H}_{\mathrm{C} 1}-\mathrm{H}_{\mathrm{C} 2}\right) / 2$ and $\mathrm{H}_{E}=-\left(\mathrm{H}_{\mathrm{C} 1}+\mathrm{H}_{\mathrm{C} 2}\right) / 2$. Here, $\mathrm{H}_{\mathrm{C} 1}$ and $\mathrm{H}_{\mathrm{C} 2}$ are the values obtained under the increasing and the decreasing magnetic field, respectively. Since the value of $\mathrm{H}_{E}$ is much greater than that of $\mathrm{H}_{C}$ in the $\mathrm{Co} / \mathrm{Ni}$ multilayer with $50 \AA \mathrm{FeRh}$, we can confirm that strong exchange interaction exists at the AFM/FM interface. In order to further investigate the AFM thickness dependent features of the exchange bias system, we produced a number of samples having different thickness of the AFM FeRh layer from $10 \AA$ to $50 \AA$ and measured the respective magnetic hysteresis loop.

As shown in Fig. 2, different hysteresis loops were obtained as the thickness of the AFM FeRh layer varies. First, the magnetic hysteresis loops of the samples show almost perfect rectangular shape, which means the present systems maintain the perpendicular magnetic anisotropy well even if the AFM FeRh layer is adjacent to the FM Co/Ni multilayer regardless of $t_{A F M}$. Interestingly, as the $t_{A F M}$ increases, the center position of the magnetic hysteresis loop placing at the origin gradually moves to the left for samples with $t_{A F M}>25 \AA$. This means the exchange bias effect appears more and more from $t_{A F M}=25 \AA$. 
For quantitative analysis, exact $\mathrm{H}_{C}$ and $\mathrm{H}_{E}$ were calculated using the relationships mentioned previously. As shown in Fig. $3, \mathrm{H}_{E}$ is almost zero in the films of $t_{A F M}<20 \AA$, however, it begins to arise from $t_{A F M}=25 \AA$ and gradually increases with thicker $t_{A F M}$. While it is predicted that the value of $\mathrm{H}_{E}$ is saturated after particular thickness $t_{A F M}=\sim 50 \AA$, this is because only the AFM layer having an effective thickness affect on the exchange bias effect in the AFM/FM structure[1,2]. Non-linear change characteristics of the coercivity $\mathrm{H}_{C}$ is known to be due to the complex interaction between the FM and AFM magnetic domains appearing at interface. However, for $\mathrm{Co} / \mathrm{Pd}$ and $\mathrm{Co} / \mathrm{Pt}$ multilayers, the change characteristics of the $\mathrm{H}_{C}$ shows monotonically increasing tendency with respect to the thickness of AFM layer as same as the $\mathrm{H}_{E}$ [9], unlike the case of the present $\mathrm{Co} / \mathrm{Ni}$ multilayer. In other words, the maximum AFM/FM magnetic domain wall interaction and the maximum $\mathrm{H}_{E}$ appear simultaneously in the same $t_{A F M}$ for the $\mathrm{Co} / \mathrm{Pd}$ and $\mathrm{Co} / \mathrm{Pt}$ multilayers, whereas the maximum domain wall interaction occurs with thinner AFM layer than $t_{A F M}$ where the $\mathrm{H}_{E}$ shows the maximum value in the present $\mathrm{Co} / \mathrm{Ni}$ multilayer system. This implies that the $\mathrm{H}_{C}$ of the magnetization inversion in the $\mathrm{Co} / \mathrm{Ni}$ multilayer is much more sensitive to the AFM/FM interface compared to the magnetization inversion of the $\mathrm{Co} / \mathrm{Pd}$ and the $\mathrm{Co} / \mathrm{Pt}$ multilayers.

In order to investigate the phenomenon of the magnetization inversion, we measured the inverted pattern of the magnetic domain in an exchange bias sample with $t_{A F M}=50 \AA$ showing an asymemetic hyteresis loop. The real-time magnetic domain revolution patterns were obtained in each region with the increasing and the decreasing magnetic field corresponding to the points of $\mathrm{H}_{C 1}$ and $\mathrm{H}_{\mathrm{C} 2}$ in Fig. 1, respectively.

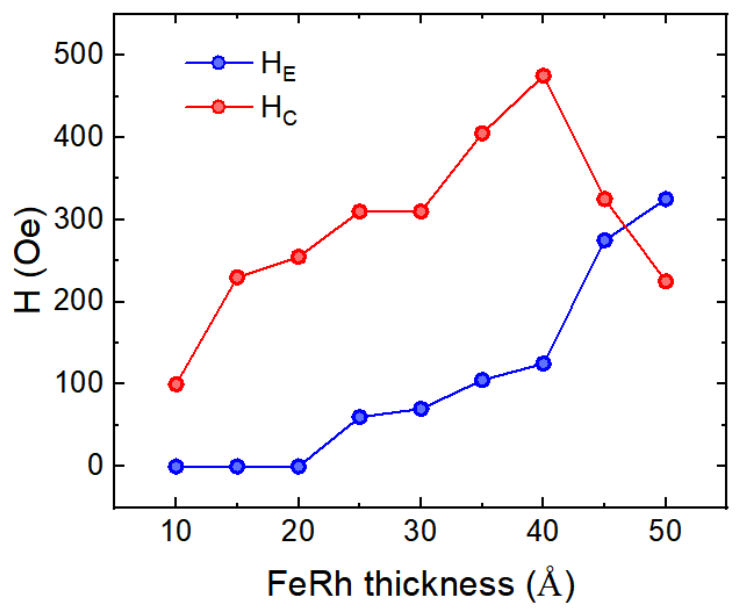

Figure 3. Dependence of the coercivity $\mathrm{H}_{C}$ and the exchange biased field $\mathrm{H}_{E}$ as a function of the AFM FeRh thickness, respectively. Here, the values of $\mathrm{H}_{C}$ and $\mathrm{H}_{E}$ were obtained using relations of $\mathrm{H}_{\mathrm{C}}=\left(\mathrm{H}_{\mathrm{C} 1} \mathrm{H}_{\mathrm{C} 2}\right) / 2$ and $\mathrm{H}_{E}=\left(\mathrm{H}_{\mathrm{C} 1}+\mathrm{H}_{\mathrm{C} 2}\right) / 2$, respectively.

As shown in Fig. 4, inversion of magnetization in regions of the $\mathrm{H}_{C 1}$ and the $\mathrm{H}_{C 2}$ reveals a tendency of the domain wall movement, and there is not notable difference between the $\mathrm{H}_{C 1}$ and the $\mathrm{H}_{\mathrm{C} 2}$ in perspective of the magnetic domain revolution pattern. Moreover, it was confirmed that only a single domain wall movement is visible in the both magnetic field area for all samples with various $t_{A F M}$. On the other hand, in the case of $\mathrm{Co} / \mathrm{Pt}$ and $\mathrm{Co} / \mathrm{Pd}$ multilayers[24,41,42], domain wall movement is completely different from the $\mathrm{Co} / \mathrm{Ni}$ multilayer. Therefore, in the case of $\mathrm{Co} / \mathrm{Ni}$ multilayer, it can be assumed that the complex interaction of the AFM/FM domain walls at the interface plays a key role rather than the asymmetric isotropic characteristics due to the exchange bias.

\section{Conclusions}

In this study, we produced exchange bias structure composed of the FM Co/Ni multilayer and the AFM FeRh layer having a perpendicular magnetic anisotropy. Changes of the exchange bias 


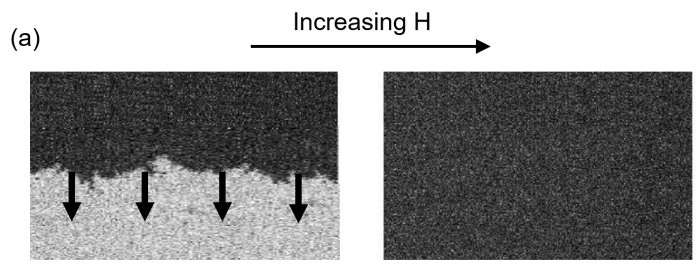

(b)

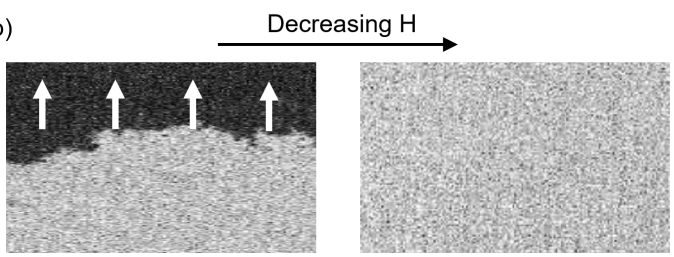

Figure 4. Domain revolution patterns with increasing $\mathrm{H}$ at $(\mathrm{a}) \mathrm{H}_{\mathrm{C} 1}$ and with decreasing $\mathrm{H}$ at $(\mathrm{b}) \mathrm{H}_{\mathrm{C} 2}$. The black and white colors represent the different magnetization directions, respectively.

magnetic field $\mathrm{H}_{E}$ and the coercivity $\mathrm{H}_{C}$ was investigated by measuring the magnetic hysteresis loops. In particular, as a thickness of the AFM FeRh increases from $10 \AA$ to $50 \AA$ the exchange bias effect was started to appear from $25 \AA$, and such effect is continued to increase up to $50 \AA$. In perspective of the pattern of magnetic domain revolution, there is not a visible difference between the regions with increasing and decreasing magnetic field for the present $\mathrm{Co} / \mathrm{Ni}$ multilayer adjacent to the AFM FeRh layer.

Funding: This research was funded by ETH Zurich central funding.

Acknowledgments: Authors gratefully acknowledge ScopeM ETH Zurich for the overall use of its facilities.

Conflicts of Interest: The authors declare no conflict of interest.

1. Nogués, J.; Schuller, I.K. Exchange bias. Journal of Magnetism and Magnetic Materials 1999, 192, 203-232.

2. Koon, N. Calculations of exchange bias in thin films with ferromagnetic/antiferromagnetic interfaces. Physical review letters 1997, 78, 4865.

3. Ohldag, H.; Scholl, A.; Nolting, F.; Arenholz, E.; Maat, S.; Young, A.; Carey, M.; Stöhr, J. Correlation between exchange bias and pinned interfacial spins. Physical review letters 2003, 91, 017203.

4. Morales, R.; Li, Z.P.; Olamit, J.; Liu, K.; Alameda, J.; Schuller, I.K. Role of the antiferromagnetic bulk spin structure on exchange bias. Physical review letters 2009, 102, 097201.

5. Nogués, J.; Lederman, D.; Moran, T.; Schuller, I.K. Positive Exchange Bias in $\mathrm{FeF}_{2}$-Fe Bilayers. Physical review letters 1996, 76, 4624.

6. Miltényi, P.; Gierlings, M.; Keller, J.; Beschoten, B.; Güntherodt, G.; Nowak, U.; Usadel, K.D. Diluted antiferromagnets in exchange bias: Proof of the domain state model. Physical Review Letters 2000, 84, 4224.

7. Mougin, A.; Mewes, T.; Jung, M.; Engel, D.; Ehresmann, A.; Schmoranzer, H.; Fassbender, J.; Hillebrands, B. Local manipulation and reversal of the exchange bias field by ion irradiation in $\mathrm{FeNi} / \mathrm{FeMn}$ double layers. Physical Review B 2001, 63, 060409.

8. Roy, S.; Fitzsimmons, M.; Park, S.; Dorn, M.; Petracic, O.; Roshchin, I.V.; Li, Z.P.; Batlle, X.; Morales, R.; Misra, A.; others. Depth profile of uncompensated spins in an exchange bias system. Physical review letters 2005, 95, 047201.

9. Sort, J.; Baltz, V.; Garcia, F.; Rodmacq, B.; Dieny, B. Tailoring perpendicular exchange bias in [Pt/Co]-IrMn multilayers. Physical Review B 2005, 71, 054411.

10. Sort, J.; Dieny, B.; Fraune, M.; Koenig, C.; Lunnebach, F.; Beschoten, B.; Güntherodt, G. Perpendicular exchange bias in antiferromagnetic-ferromagnetic nanostructures. Applied physics letters 2004, 84, 3696-3698. 
11. Garcia, F.; Sort, J.; Rodmacq, B.; Auffret, S.; Dieny, B. Large anomalous enhancement of perpendicular exchange bias by introduction of a nonmagnetic spacer between the ferromagnetic and antiferromagnetic layers. Applied physics letters 2003, 83, 3537-3539.

12. Zhou, S.; Sun, L.; Searson, P.; Chien, C. Perpendicular exchange bias and magnetic anisotropy in CoO/permalloy multilayers. Physical Review B 2004, 69, 024408.

13. Radu, F.; Abrudan, R.; Radu, I.; Schmitz, D.; Zabel, H. Perpendicular exchange bias in ferrimagnetic spin valves. Nature Communications 2012, 3, 715.

14. Fitzsimmons, M.; Leighton, C.; Nogués, J.; Hoffmann, A.; Liu, K.; Majkrzak, C.; Dura, J.; Groves, J.; Springer, R.; Arendt, P.; others. Influence of in-plane crystalline quality of an antiferromagnet on perpendicular exchange coupling and exchange bias. Physical review B 2002, 65, 134436.

15. van Dijken, S.; Moritz, J.; Coey, J. Correlation between perpendicular exchange bias and magnetic anisotropy in $\mathrm{IrMn} /[\mathrm{Co} / \mathrm{Pt}]_{n}$ and $[\mathrm{Pt} / \mathrm{Co}]_{n} / \mathrm{IrMn}$ multilayers. Journal of applied physics 2005, 97, 063907.

16. Echtenkamp, W.; Binek, C. Electric control of exchange bias training. Physical review letters 2013, 111, 187204.

17. He, X.; Wang, Y.; Wu, N.; Caruso, A.N.; Vescovo, E.; Belashchenko, K.D.; Dowben, P.A.; Binek, C. Robust isothermal electric control of exchange bias at room temperature. Nature materials 2010, 9, 579.

18. Egelhoff Jr, W.F.; McMichael, R.D.; Mallett, J.; Shapiro, A.J.; Powell, C.J.; Bonevich, J.E.; Judy, J.; Thomas, J.; Svedberg, E.B. Origin of exchange decoupling effects in high-coercivity air-annealed CoPd multilayers. Journal of applied physics 2005, 97, $10 \mathrm{~J} 104$.

19. Suzuki, I.; Hamasaki, Y.; Itoh, M.; Taniyama, T. Controllable exchange bias in Fe/metamagnetic FeRh bilayers. Applied Physics Letters 2014, 105, 172401.

20. Nam, N.T.; Lu, W.; Suzuki, T. Exchange bias of ferromagnetic/antiferromagnetic in FePt/FeRh bilayers. Journal of Applied Physics 2009, 105, 07 D708.

21. Jia, Z; Misra, R. Simulated temperature dependence of exchange bias field in exchange coupled FeRh/FePt bilayer: relevance in magnetic recording media. Materials Technology 2010, 25, 307-312.

22. Marrows, C. Three-dimensional exchange bias in $\{\mathrm{Co} / \mathrm{Pd}\}_{N} /$ FeMn. Physical review B 2003, $68,012405$.

23. Romanens, F.; Pizzini, S.; Yokaichiya, F.; Bonfim, M.; Pennec, Y.; Camarero, J.; Vogel, J.; Sort, J.; Garcia, F.; Rodmacq, B.; others. Magnetic relaxation of exchange biased Pt/Co multilayers studied by time-resolved Kerr microscopy. Physical Review B 2005, 72, 134410.

24. Czapkiewicz, M.; Stobiecki, T.; van Dijken, S. Thermally activated magnetization reversal in exchange-biased [Pt/ Co $]_{3} / \mathrm{Pt} / \mathrm{IrMn}$ multilayers. Physical Review B 2008, 77, 024416.

25. Kagerer, B.; Binek, C.; Kleemann, W. Freezing field dependence of the exchange bias in uniaxial $\mathrm{FeF}_{2}-\mathrm{CoPt}_{\mathrm{H}}$ heterosystems with perpendicular anisotropy. Journal of magnetism and magnetic materials 2000, 217, 139-146.

26. Toyoki, K.; Shiratsuchi, Y.; Kobane, A.; Mitsumata, C.; Kotani, Y.; Nakamura, T.; Nakatani, R. Magnetoelectric switching of perpendicular exchange bias in $\mathrm{Pt} / \mathrm{Co} / \alpha-\mathrm{Cr} 2 \mathrm{O} 3 / \mathrm{Pt}$ stacked films. Applied Physics Letters 2015, 106, 162404.

27. Ehresmann, A.; Lengemann, D.; Weis, T.; Albrecht, A.; Langfahl-Klabes, J.; Göllner, F.; Engel, D. Asymmetric Magnetization Reversal of Stripe-Patterned Exchange Bias Layer Systems for Controlled Magnetic Particle Transport. Advanced Materials 2011, 23, 5568-5573.

28. Stamps, R. Mechanisms for exchange bias. Journal of Physics D: Applied Physics 2000, 33, R247.

29. Kiwi, M. Exchange bias theory. Journal of Magnetism and Magnetic materials 2001, 234, 584-595.

30. Mangin, S.; Ravelosona, D.; Katine, J.; Carey, M.; Terris, B.; Fullerton, E.E. Current-induced magnetization reversal in nanopillars with perpendicular anisotropy. Nature materials 2006, 5, 210.

31. Tanigawa, H.; Koyama, T.; Yamada, G.; Chiba, D.; Kasai, S.; Fukami, S.; Suzuki, T.; Ohshima, N.; Ishiwata, N.; Nakatani, Y.; others. Domain wall motion induced by electric current in a perpendicularly magnetized Co/Ni nano-wire. Applied Physics Express 2009, 2, 053002.

32. Ryu, K.S.; Thomas, L.; Yang, S.H.; Parkin, S.S. Current induced tilting of domain walls in high velocity motion along perpendicularly magnetized micron-sized Co/Ni/Co racetracks. Applied Physics Express 2012, 5, 093006.

33. Koyama, T.; Hata, H.; Kim, K.J.; Moriyama, T.; Tanigawa, H.; Suzuki, T.; Nakatani, Y.; Chiba, D.; Ono, T. Current-induced magnetic domain wall motion in a $\mathrm{Co} / \mathrm{Ni}$ nanowire with structural inversion asymmetry. Applied Physics Express 2013, 6, 033001. 
34. Chiba, D.; Yamada, G.; Koyama, T.; Ueda, K.; Tanigawa, H.; Fukami, S.; Suzuki, T.; Ohshima, N.; Ishiwata, N.; Nakatani, Y.; others. Control of multiple magnetic domain walls by current in a Co/Ni nano-wire. Applied Physics Express 2010, 3, 073004.

35. Fukami, S.; Suzuki, T.; Tanigawa, H.; Ohshima, N.; Ishiwata, N. Stack structure dependence of Co/Ni multilayer for current-induced domain wall motion. Applied physics express 2010, 3, 113002.

36. Yoshimura, Y.; Koyama, T.; Chiba, D.; Nakatani, Y.; Fukami, S.; Yamanouchi, M.; Ohno, H.; Ono, T. Current-induced domain wall motion in perpendicularly magnetized $\mathrm{Co} / \mathrm{Ni}$ nanowire under in-plane magnetic fields. Applied Physics Express 2012, 5, 063001.

37. Mohseni, S.M.; Yazdi, H.; Hamdi, M.; Brächer, T.; Mohseni, S.M. Current induced multi-mode propagating spin waves in a spin transfer torque nano-contact with strong perpendicular magnetic anisotropy. Journal of Magnetism and Magnetic Materials 2018, 450, 40-45.

38. Tang, M.; Zhao, B.; Zhu, W.; Zhu, Z.; Jin, Q.; Zhang, Z. Controllable Interfacial Coupling Effects on the Magnetic Dynamic Properties of Perpendicular [Co/Ni] 5/Cu/TbCo Composite Thin Films. ACS applied materials $\mathcal{E}$ interfaces 2018, 10, 5090-5098.

39. Kolesnikov, A.; Wu, H.; Stebliy, M.; Ognev, A.; Chebotkevich, L.; Samardak, A.; Han, X. Hybrid magnetic anisotropy [Co/Ni] 15/Cu/[Co/Pt] 4 spin-valves. Journal of Magnetism and Magnetic Materials 2018, 449, 271-277.

40. Jekal, S.; Rhim, S.; Hong, S.C.; Son, W.j.; Shick, A.B. Surface-termination-dependent magnetism and strong perpendicular magnetocrystalline anisotropy of an FeRh (001) thin film. Physical Review B 2015, 92, 064410.

41. Choe, S.B.; Shin, S.C. Magnetization reversal in nanostructured Co/Pd multilayers. Physical Review B 1998, $57,1085$.

42. Choe, S.B.; Shin, S.C. Observation of unequal activation volumes of wall-motion and nucleation processes in Co/Pd multilayers. Physical review letters 2001, 86, 532. 